\title{
Cardiovascular risk factors, socioeconomic determinants and angiographic severity of coronary artery disease in patients awaiting coronary artery bypass graft in a provincial hospital Sri Lanka
}

\author{
Wickramatilake CM ${ }^{1}$, Withanawasam BPS ${ }^{2}$, Mohideen MR $^{3}$ \\ ${ }^{\prime}$ Department of Biochemistry, Faculty of Medicine, University of Ruhuna, Galle, Sri Lanka \\ ${ }^{2}$ Institute of Cardiology, National Hospital, Colombo, Sri Lanka \\ ${ }^{3}$ Department of Medicine, Faculty of Medicine, University of Ruhuna, Galle, Sri Lanka
}

Correspondence: Dr. C.M. Wickramatilake

e-mail: chandimadhu@live.com

\begin{abstract}
Introduction: The age-standardized death rate of coronary heart disease has shown a downward trend in many developed countries while an upward trend has been seen in developing countries with demographic changes, urbanization, and lifestyle changes. The aims of our study were to study the prevalence of cardiovascular risk factors (CVRFs), socioeconomic determinants and explore the relationship between CVRFs and angiographic severity of coronary artery disease (CAD) in a cohort of patients awaiting coronary artery bypass graft (CABG).

Methods: It was a cross-sectional descriptive study. One hundred and forty one consecutive male patients with angiographically diagnosed CAD were selected. We examined the relationship between traditional CVRFs and atherosclerotic burden assessed by different vessel scores.

Results: Mean (SD) age of the subjects was 56(8) years. The prevalence of CVRFs was high in the study group. Majority of them were in the lower socioeconomic status (SES). The analysis indicated that there were no significant correlations or a significant predictive value of CVRFs with the severity ofCAD.

Conclusions: Severity of coronary artery disease estimated by angiographic scores appears to correlate poorly with established traditional cardiovascular risk factors.
\end{abstract}

Key words: Cardiovascular risk factors, Socioeconomic determinants, Angiographic severity of coronary artery disease.

\section{Introduction}

The age-standardized death rate of coronary heart disease has shown a downward trend in many developed countries while an upward trend has been seen in developing countries such as Sri Lanka with demographic changes, urbanization, and lifestyle changes $(1,2)$. Coronary artery disease $(\mathrm{CAD})$ is one of the leading causes of mortality in men. Traditional cardiovascular risk factors (CVRFs) such as advancing age, diabetes mellitus, hypertension, dyslipidaemia, smoking, obesity, and family history of CAD are well recognized for their association as risk factors with $\mathrm{CAD}(3)$. However, the correlation between CVRFs and atherosclerotic burden, assessed angiographically is not as well established, reporting variable and inconsistent results (4-11).

The aims of our study were to characterize socioeconomic determinants, CVRFs and explore the relationship between CVRFs and angiographic severity of CAD in a cohort of patients awaiting coronary artery bypass graft. 


\section{Methods}

This cross sectional descriptive study investigated 141 consecutive male patients (age 35-76 years) with angiographically diagnosed $\mathrm{CAD}$, awaiting CABG in the Cardiothoracic Unit, Teaching Hospital Karapitiya, Sri Lanka.

An interviewer-administered questionnaire was used in the collection of data. The following information was gathered during the interview and examination: socio-demographic data, presence of traditional cardiovascular risk factors. The socioeconomic status (SES) was defined by occupational position, education and income according to the Barker \& Hall guidelines (12).

All baseline anthropometric measurements were estimated by the same investigator, using same instruments. Height was measured following standard technique by a portable stadiometer (IUCHI, Yamato Scientific, Japan) with the precision of $+/-0.1 \mathrm{~cm}$ and readability up to $200 \mathrm{~cm}$. Weight was measured using a portable beam balance (Bauman, Germany) with the precision of $+/-0.1 \mathrm{~kg}$ and readability up to $100 \mathrm{~kg}$. Waist circumference was measured to the nearest $0.1 \mathrm{~cm}$ according to the standard technique using a non-stretchable measuring tape. Mean of the two readings (three times if difference between readings was $\geq 0.5 \mathrm{~cm}$ ) was taken as the final measurement. Body mass index (BMI) was calculated.

The interpretation and grades of luminal narrowing were determined according to the consensus opinion of two separately read angiography reports by interventional cardiologists who were blinded for the characteristics of the patients. Three methods were employed in the assessment of the severity of the CAD; Gensini score (13), Leaman score (14), vessel score (15).

This clinical protocol was approved by the Ethical Review Committee of Faculty of Medicine, University of Ruhuna, Galle, Sri Lanka and conducted according to the ethical guidelines outlined in the Declaration of Helsinki. Permission from the respective hospital authorities was obtained. Informed written consent was given by all the participants.

Data were analyzed using Minitab version 15. Results were expressed as mean (standard deviation), percentages or frequencies. Statistical significance was defined when $P$ value was $<0 \cdot 05$. Coronary vessel score was divided into four groups as zero, one, two and three-vessel disease. Gensini and Leaman scores were categorized into four categories according to the quartiles ranges. Ordinal logistic regression analysis was performed to determine the predictors of the extent of CAD (on the scale three scores used).

\section{Results}

A total of 141 male patients with $\mathrm{CAD}$ were included. Mean (SD) age of subjects was 56 (8) years (range: $35-76$ years). Table 1 shows the prevalence of cardiovascular risk factors, demographic and socioeconomic characteristics of the patients. Body mass index (BMI) ranged from $17.8 \mathrm{kgm}^{-2}$ to $38 \mathrm{kgm}^{-}$ ${ }^{2}$ and the mean (SD) was $24(3) \mathrm{kgm}^{-2}$. Majority of patients $(66.7 \%)$ were within the normal BMI limits and only $31.9 \%$ were in the overweight and obesity groups, according to the WHO criteria (16). The prevalence of overweight and obesity $(60.2 \%)$ was higher with the use of lower cut off values $(\geq 23 \mathrm{kgm}$ ${ }^{2}$ as over weight) defined for the Asian populations by International Association for the Study of Obesity (IASO), and the International Obesity Task Force (IOTF) (17).

Table 1: Basic clinical, demographic and socioeconomic characteristics $(\mathrm{n}=141)$

\begin{tabular}{lc}
\hline Characteristics & Mean (SD) or n (\%) \\
\hline Age (years) & $56 \pm 8$ \\
BMI $\left(\mathrm{kgm}^{-2}\right)$ & $24 \pm 3$ \\
Obesity $\left(\mathrm{BMI}>30 \mathrm{kgm}^{-2}\right)$ & $5(3.6 \%)$ \\
Diabetes mellitus & $56(39.7 \%)$ \\
Dyslipidaemia & $50(35.5 \%)$ \\
Hypertension & $67(47.5 \%)$ \\
Smoking & $105(74.5 \%)$ \\
Alcohol intake & $107(75.9 \%)$ \\
Statin treatment & $136(96.4 \%)$ \\
\hline
\end{tabular}

\section{Social classes}

Class 1 $10(7.1 \%)$

Class 2

$36(25.5 \%)$

Class 3

$37(26.2 \%)$

Class 4

$41(29.1 \%)$

Class 5

$17(12.1 \%)$

$\mathrm{A} / \mathrm{L}=$ Advanced level examination. $\mathrm{O} / \mathrm{L}=$ Ordinary level examination 
Waist circumference varied from $70.2 \mathrm{~cm}$ to 115.5 $\mathrm{cm}$ with a mean and SD of 87.1 and $7.6 \mathrm{~cm}$. Minority of the patients $(17.7 \%)$ had a waist circumference above the cut off value defined for males $(\geq 94 \mathrm{~cm})$, that indicates the central obesity by the WHO criteria, but when Asian population specific cutoff values for WC $(\geq 90 \mathrm{~cm})$ was used it rose to $28.3 \%$.

The vessel score varied from zero to three, Gensini score ranged from zero to 166 and Leaman score varied from zero to 35 . Table 2 indicates the severity categories according to the three scores and the percentage of patients in each category. The other tables (Table 3, 4, 5) demonstrate the significant values, odd ratios and the relevant confidence intervals for each predictor variables considered for atherosclerotic burden categories following ordinal logistic regression. The results reflected that the traditional cardiovascular risk factors were not independent predictors of severity of CAD.
Table 2: Angiographic characteristics of the study $\operatorname{cohort}(\mathrm{n}=141)$

\begin{tabular}{cc}
\hline Angiographic findings & Number (\%) \\
\hline Vessel score categories & $2(1.4 \%)$ \\
Minor vessel disease (zero) & $8(5.7 \%)$ \\
One vessel disease & $29(20.6 \%)$ \\
Two vessel disease & $12(72.3 \%)$ \\
Three vessel disease & \\
\hline Gensini score quartiles & $36(25.5 \%)$ \\
$0-50$ & $37(26.2 \%)$ \\
$>50-72$ & $33(23.4 \%)$ \\
$>72-98.5$ & $35(24.8 \%)$ \\
$>98.5-166$ & \\
\hline Leaman score quartiles & $36(25.5 \%)$ \\
$0-9.5$ & $36(25.5 \%)$ \\
$>9.5-15$ & $37(26.2 \%)$ \\
$>15-20$ & $32(22.7 \%)$ \\
\hline
\end{tabular}

Table 3: Predictors of atherosclerotic burden assessed by Gensini score among patients with CAD ( $\mathrm{n}=141)$

\begin{tabular}{lccc}
\hline & Odd ratio & $\mathbf{9 5} \%$ CI for $\boldsymbol{\beta}$ & P value \\
\hline Age (1- year increase) & 0.99 & $0.96-1.03$ & 0.666 \\
Obesity (BMI $\left.>30 \mathrm{kgm}^{-2}\right)$ & 0.67 & $0.13-3.52$ & 0.637 \\
Diabetes mellitus & 1.37 & $0.74-2.54$ & 0.312 \\
Hypertension & 0.45 & $0.24-0.84$ & 0.012 \\
Smoking & 1.40 & $0.67-2.92$ & 0.369 \\
Alcohol intake & 0.90 & $0.43-1.89$ & 0.782 \\
Dyslipidaemia & 1.28 & $0.67-2.47$ & 0.451 \\
\hline
\end{tabular}

Results of the ordinal logistic regression analysis are given as odd ratio (OR) and confidence intervals (CI).

Table 4: Predictors of atherosclerotic burden assessed by Leaman score among patients with CAD $(n=141)$

\begin{tabular}{lccc}
\hline & Odd ratio & $\mathbf{9 5} \%$ CI for $\boldsymbol{\beta}$ & P value \\
\hline Age (1-year increase) & 0.98 & $0.95-1.02$ & 0.311 \\
Obesity $\left(\mathrm{BMI}>30 \mathrm{kgm}^{-2}\right)$ & 0.37 & $0.07-2.01$ & 0.251 \\
Diabetes mellitus & 1.21 & $0.66-2.23$ & 0.541 \\
Hypertension & 0.62 & $0.33-1.15$ & 0.126 \\
Smoking & 0.98 & $0.47-2.05$ & 0.966 \\
Alcohol intake & 0.84 & $0.40-1.77$ & 0.654 \\
Dyslipidaemia & 0.93 & $0.49-1.79$ & 0.835 \\
\hline
\end{tabular}

Results of the ordinal logistic regression analysis are given as odd ratio (OR) and confidence intervals. 
Table 5: Predictors of atherosclerotic burden assessed by vessel score among patients with CAD ( $\mathrm{n}=141)$

\begin{tabular}{lccc}
\hline & Odd ratio & $\mathbf{9 5} \%$ CI for $\boldsymbol{\beta}$ & P value \\
\hline Age (1 year increase) & 1.01 & $0.97-1.06$ & 0.598 \\
Obesity $\left(\mathrm{BMI}>30 \mathrm{kgm}^{-2}\right)$ & 0.70 & $0.07-7.28$ & 0.765 \\
Diabetes mellitus & 0.37 & $0.16-0.86$ & 0.020 \\
Hypertension & 0.81 & $0.37-1.78$ & 0.606 \\
Smoking & 1.62 & $0.66-3.96$ & 0.289 \\
Alcohol intake & 1.70 & $0.64-4.53$ & 0.284 \\
Dyslipidaemia & 0.54 & $0.22-1.28$ & 0.161 \\
\hline
\end{tabular}

Results of the ordinal logistic regression analysis are given as odd ratio (OR) and confidence intervals.

\section{Discussion}

Our findings suggest that majority of patients with CAD undergoing coronary artery bypass graft in a local provincial hospital belongs to lower socioeconomic classes. Prevalence of traditional risk factors was high among these patients. The anthropometric parameters did not appear to predict the atherosclerotic burden assessed by angiogram based tools. Diabetes mellitus was found to be an independent predictor of the atherosclerotic burden estimated by the vessel score, but OR was less than 1 . Hypertension seemed to be an independent predictor of the severity of CAD assessed by the Gensini score with a low odds ratio. Therefore none of the traditional risk factors emerged as a meaningful independent predictor of atherosclerotic burden. The associations of CVRFs with coronary atherosclerotic burden have been variable and inconsistencies exist. Few have shown positive associations, while others have reported equivocal or even negative associations (4-11, 18).

Wang et al. found that the total amount of lifetime smoking and total cholesterol/ HDL-cholesterol was associated with the severity of coronary artery disease (4). Phillips et al., showed that in men selected for coronary arteriography; age and HDL-cholesterol might be stronger predictors of the degree of CAD than were blood pressure, cholesterol, diabetes, smoking, and BMI (5). Vlietstra et al. concluded that some risk factors were associated with the presence of disease and also with the extent of the CAD, but other factors appeared to influence only to the onset of the disease (6). Anderson et al. also showed a variable relationship between cardiovascular risk factors and the extent of coronary artery disease (7). According to Hasin et al. the most important contributory factors to the severity of atherosclerosis were the duration of clinical history, number of previous myocardial infarctions, and male sex, but more specifically elevation of serum cholesterol and diabetes mellitus. Cigarette smoking, obesity, hypertension, a family history of atherosclerosis, and elevated serum triglycerides had a positive influence, but this was not statistically significant (8). Krishna Swami et al., revealed that although strong associations exist between risk factors and the occurrence of CAD, the small quantitative association detected between the presence of risk factors and the severity of disease was weak (9). Opherk et al., found a significant correlation between the degree of atherosclerotic lesions and hypertriglyceridaemia, hypercholesterolaemia as well as smoking habits. No correlation between other risk factors, such as hypertension, diabetes mellitus, hyperuricaemia, obesity, and the coronary score was observed (10). Guo et al., demonstrated high low-density lipoprotein as a prominent predictor of the extent and severity of angiographic CAD (11). Nicholls et al. showed male gender, diabetes mellitus, and a history of prior revascularization were as strong independent predictors of atherosclerotic burden in coronary disease patients. However many risk 
factors did not predict angiographic disease severity, suggesting different mechanisms drive stenosis development and atheroma accumulation (19).

Similarly, in a study involving younger patients (mean age 56 years) to determine the relationship between CVRFs and the extent of CAD, only a few risk factors (diabetes and male sex) correlated with intravascular ultrasonography (IVUS)measured coronary atheroma burden; none of these risk factors correlated with "luminal" severity assessed through coronary angiography, suggesting that different mechanisms drive stenosis development and atheroma accumulation (18).

Hypercholesterolaemia has been reported as an independent risk factor for severity of coronary artery disease $(8,10)$. In the present study, a trend of dyslipidaemia and higher prevalence of statin use were observed, but dyslipidemia was not a predictor of the extent of obstructive CAD burden. Similarly, a lack of association between lipids and atheroma burden, as estimated by IVUS in a recent study, prompted the speculation of other factors such as inflammation and genetic susceptibility may predict cardiovascular outcomes in the setting of dyslipidaemia (18).

In conclusion, the patients who were admitted for coronary artery bypass graft in a state local provincial hospital were mainly in the lower socioeconomic status. The conventional cardiovascular risk factors were not able to predict the severity of coronary artery disease estimated by coronary angiography based scores, although they were considerably prevalent.

The limitations we encountered were the missing of patients who were being treated at the private sector hospitals who may be included in the higher SES. The study was confined to men and need to be expanded to women.

\section{Disclosure of interest}

The authors declare that they have no conflicts of interest concerning this article.

\section{Acknowledgement}

We wish to acknowledge the University Grants Commission, Sri Lanka for the financial assistance provided for the project (Grant No: UGC/ICD/CRF/ 2009/2/47) and Mrs. D.A.B.N. Amerasekara (Statistician, Applied Statistic Association, Sri Lanka), Lecturer, Department of Crop Science, Faculty of Agriculture, University of Ruhuna, Sri Lanka for the statistical support provided, Dr. N. Gamage and staff, Cardiothoracic Unit, Teaching Hospital, Karapitiya, Galle for the cooperation provided for the project.

\section{Financial support information}

University Grants Commission, Sri Lanka provided the financial assistance for the project (UGC/ICD/CRF/ 2009/2/47).

\section{References}

1. Rosamond W, Flegal K, Furie K, et al. Heart disease and stroke statistics - 2008 update: A report from the American Heart Association Statistics Committee and Stroke Statistics Subcommittee. Circulation 2008; 117: e25e146.

2. Annual Health Bulletin, Ministry of Health, Sri Lanka 2007: 31 .

3. Yusuf S, Hawken S, Ounpuu S, et al. Effect of potentially modifiable risk factors associated with myocardial infarction in 52 countries (the INTERHEART study): case-control study. Lancet 2004; 364: 937-52.

4. Wang XL, Tam C, McCredie RM, Wilcken DE. Determinants of severity of coronary artery disease in Australian men and women. Circulation 1994; 89:1974-81.

5. Phillips GB, Pinkernell BH, Jing TY. Are major risk factors for myocardial infarction the major predictors of degree of coronary artery disease in men? Metabolism 2004; 53: 324-9.

6. Vlietstra RE, Kronmal RA, Frye RL, et al. Factors affecting the extent and severity of coronary artery disease in patients enrolled in the coronary artery surgery study. Arteriosclerosis 1982; 2: 208-15.

7. Anderson AJ, Barboriak JJ, Rimm AA. Risk factors and angiographically determined coronary occlusion. American Journal of Epidemiology 1978; 107: 8-14.

8. Hasin Y, Eisenberg S, Friedlander J, et al. Relationship between extent of coronary artery disease and correlative risk factors. American Heart Journal 1979; 98: 555-61.

9. Krishnaswami S, Jose VJ, Joseph G. Lack of correlation between coronary risk factors and CAD severity. International Journal of Cardiology 1994; 47: 37-43. 
10. Opherk D, Scola R, Morgenstern W, et al. Coronary risk factors and extent of angiographically proven coronary artery stenoses (author's transl). Zeitschrift für Kardiologie 1979; 68: 369-74.

11. Guo YH, Zhang WJ, Zhou YJ, et al. Study of the relationship between cardiovascular risk factors and severity of coronary artery disease in patients underwent coronary angiography. Zhonghua Xin Xue Guan Bing Za Zhi 2005; 33: 415-8.

12. Barker DJP, Hall AJ. Practical Epidemiology $4^{\text {th }}$ ed. 1992; 65-8.

13. Gensini GG. A more meaningful scoring system for determinating the severity of coronary heart disease. American Journal of Cardiology 1983; 51: 606.

14. Leaman DM, Brower RW, Meester GT, et al. Coronary artery atherosclerosis: Severity of the disease, severity of angina pectoris and compromised left ventricular function. Circulation 1981; 63: 285-99.

15. Ringqvist I, Fisher LD, Mock M, et al.Prognostic value of angiographic indices of coronary artery disease from the Coronary Artery Surgery Study (CASS). Journal of Clinical Investigations 1983; 71: 1854-66.
16. World health organization, 1998. Obesity: Preventing and Managing the Global Epidemic. Geneva, WHO. Available at http://whqlibdoc.who.int/hq/1998/WHO_NUT_NCD_98. [Accessed 23 December 2012].

17. International Association for the Study of Obesity (IASO) and International Obesity Task Force (IOTF), 2000.The Asia Pacific Perspective; Redefining obesity and its treatment, WHO Western Pacific Region 2000. Available at http://www.iaso.org/.../IOTF_Strategic_Plan. [Accessed 13 May 2013].

18. Nicholls SJ, Tuzcu EM, Crowe T, et al. Relationship between cardiovascular risk factors and atherosclerotic disease burden measured by intravascular ultrasound. Journal of the American College of Cardiology 2006; 47: 1967-75. 